\title{
Applications des électrodes volumiques à divers problèmes électrochimiques
}

\author{
Rolando Alvarez, Manuel Fragnière, Aniko Lecoultre, François Pasquini et \\ Paul Tissot*
}

Abstract. The specific surface area of several porous electrodes has been determined by an electrochemical method, which yields similar results as the pressure drop method. Modified reticulated vitreous $\mathrm{C}$ has been applied to the electrochemical reduction of $\mathrm{O}_{2}$ to $\mathrm{H}_{2} \mathrm{O}_{2}$. Detoxification of waste water containing cyanid or $\mathrm{Ni}$ at low concentration is described.

\section{Introduction}

Le flux d'électrons qui circule entre une électrode et un électrolyte peut être limité par des phénomènes cinétiques liés à la thermodynamique, ou par des phénomènes de transfert de matière. Dans le cas d'applications électrochimiques industrielles, dans lesquelles l'aspect économique joue un rôle essentiel, on essaye de travailler avec un flux d'électrons maximum; ce flux, pour une réaction donnée, est limité par le transfert de matière, et il est mesuré par le courant limite $I_{\mathrm{L}}$ indépendant du potentiel de l'électrode (éqn. I) [1][2]

$I_{\mathrm{L}}=n F A K_{d} c_{\mathrm{O}}$

La concentration $c_{O}$, qui doit être la plus élevée possible, est en général limitée par les propriétés physico-chimiques du système. Une agitation efficace de l'électrolyte assure une valeur élevée du coefficient de transfert $K_{\mathrm{d}}$, en réduisant l'épaisseur de la couche limite de diffusion. Quant à l'aire de l'électrode par rapport au volume du réacteur électrochimique, elle peut être considérablement augmentée par l'utilisation d'électrodes volumiques tridimensionnelles à la place des électrodes bidimensionnelles classiques constituées par des plaques.
Une électrode est appelée volumique lorsque son aire accessible pour le transfert des électrons est plus grande que son aire géométrique. Il en existe plusieurs types: grilles, particules empilées ou fluidisées, feutres, mousses, etc. Les électrodes décrites dans ce travail sont des mousses rigides de différentes natures [3]:

\section{Ni 20 ppi: mousses de Ni}

PU 20 ppi: mousse de polyuréthane recouverte de $\mathrm{Ni}$

RVC 30 ppi: mousse de C vitreux

$\mathrm{PbO}_{2} 30$ ppi: mousse de $\mathrm{C}$ vitreux recouverte de $\mathrm{PbO}_{2}$
*Correspondance: Prof. P. Tissot Département de Chimie minérale, analytique et appliquée

Université de Genève, Sciences II

30, quai Ernest-Ansermet

$\mathrm{CH}-1211$ Genève 4

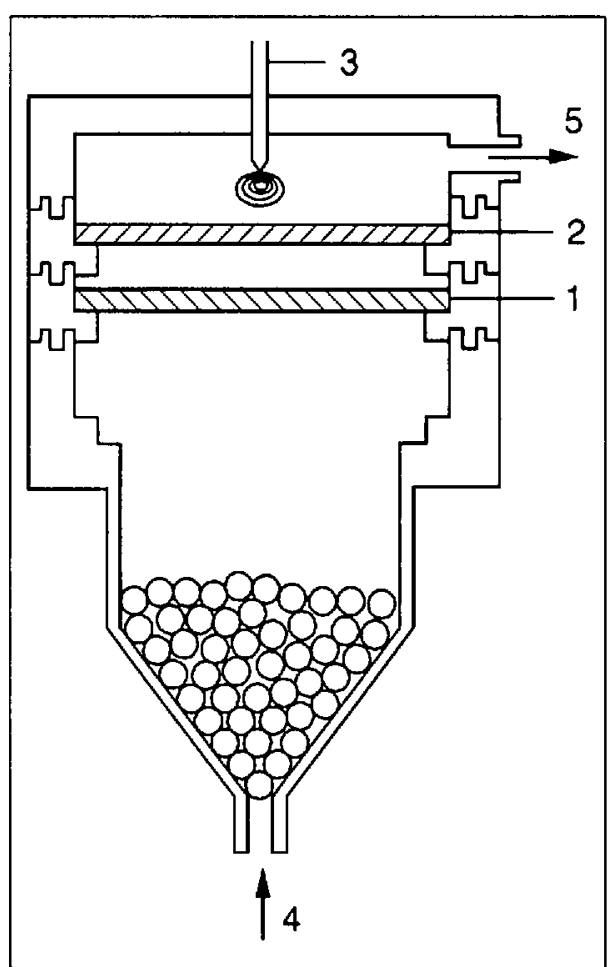

Fig. 1. Schéma de la cellule utilisée pour la détermination de $\mathrm{K}_{d} \mathrm{~A}_{e}$. l: électrode volumique; 2:contre électrode; 3 : électrode de comparaison; 4 et 5 : entrée et sortie de l'électrolyte.

La porosité de la mousse est exprimée en ppi (pores par pouce linéraire).

\section{Mesure de l'aire spécifique}

La grandeur de l'aire spécifique d'une électrode volumique, notée $A_{e}$, dépend de la méthode mise en oeuvre pour sa détermination [4][5]. Nous avons utilisé dans ce travail une méthode dynamique, qui en

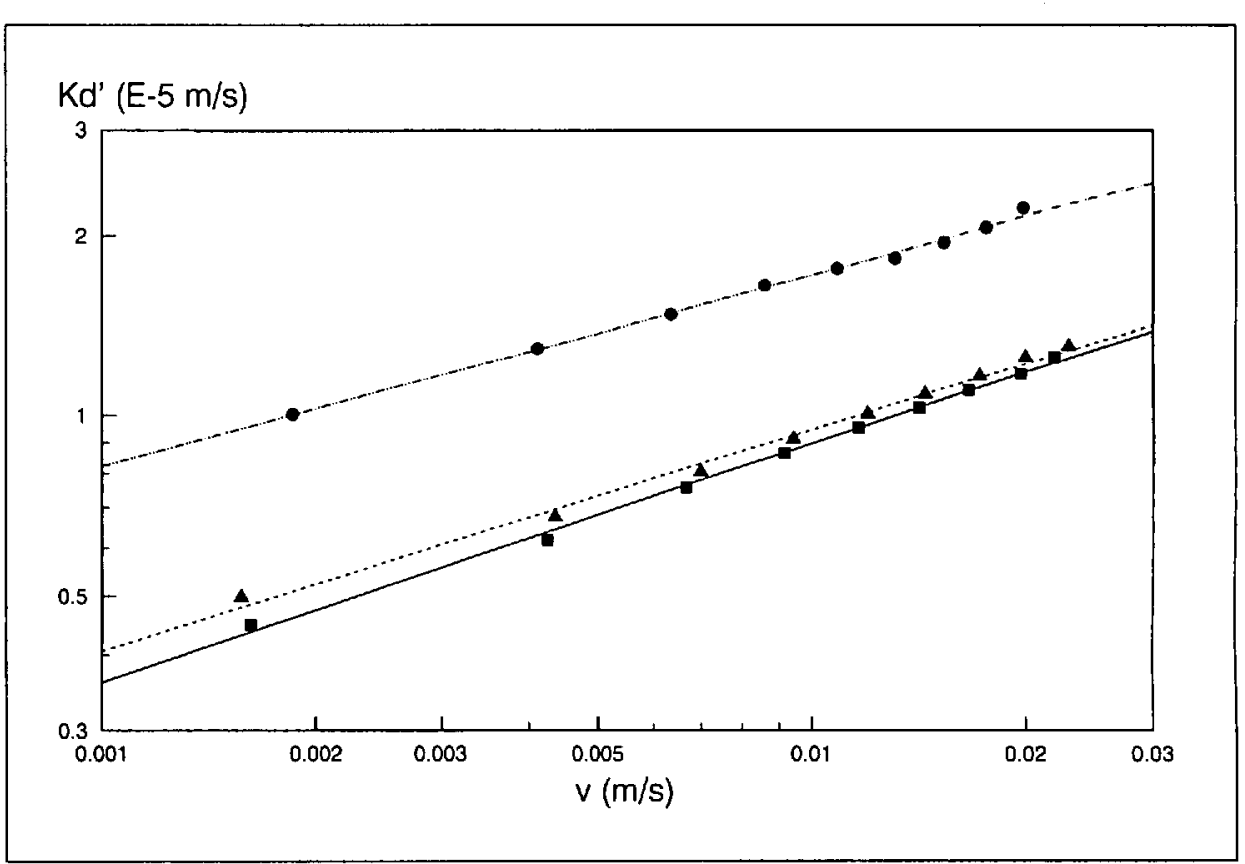

Fig. 2. Coefficient de transfert des électrodes de comparaison en fonction de la vitesse del'électrolyte.

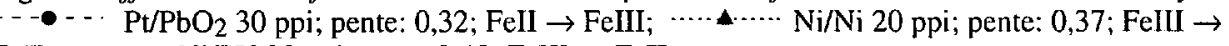
FeII; $\longrightarrow$ Ni/PU 20 ppi; pente 0,$40 ;$ FeIII $\rightarrow$ FeII. 
principe permet de mesurer l'aire électrochimiquement active; elle consiste en la détermination simultanée du courant limite sur une électrode de comparaison d'aire connue et sur une électrode volumique d'aire inconnue. La mesure est effectuée pour une même réaction électrochimique, dans des conditions identiques. Au moyen de l'éqn. 1), on détermine le coefficient de transfert $K_{d^{\prime}}$ pour l'électrode de comparaison.

Dans le cas d'un réacteur à écoulement piston, le produit $K_{\mathrm{d}} A_{\mathrm{e}}$ d'une électrode volumique peut être déterminé selon l'éqn. 2) $[1][2]$

$K_{\mathrm{d}} A_{\mathrm{c}}=\frac{V}{L} \ln \left(1-\frac{I_{\mathrm{L}}}{n \mathrm{~F} c_{\mathrm{O}} Q_{v}}\right)$

Le rapport $\frac{K_{\mathrm{d}} A_{\mathrm{e}}}{K_{\mathrm{d}^{\prime}}}$ permet d'obtenir l'aire

spécifique de l'électrode volumique, à condition que $K_{\mathrm{d}}$ et $K_{\mathrm{d}}$ soient égaux.

\subsection{Partie expérimentale}

Nous avons utilisé une cellule cylindrique de $6 \mathrm{~cm}$ de diamètre, schématiquement représentée sur la fig. $l$.

Nous avons mesuré le courant limite de l'électrode volumique et de l'électrode de comparaison à $25^{\circ}$, pour différentes vitesses de circulation de l'électrolyte, dont la composition était la suivante: $a)$ Réduction de $\mathrm{Fe}(\mathrm{CN})_{6}^{-3}$ sur PU 20 ppi et $\mathrm{Ni}$ 20 ppi: $\mathrm{NaOH} 2 \mathrm{M} ; \mathrm{K}_{3}\left[\mathrm{Fe}(\mathrm{CN})_{6}\right] 5 \cdot 10^{-3} \mathrm{M}$; $\left.\mathrm{K}_{4}\left[\mathrm{Fe}(\mathrm{CN})_{6}\right] 5 \cdot 10^{-2} \mathrm{M} ; b\right)$ Oxydation de $\mathrm{Fe}(\mathrm{CN})_{6}^{-4}$ sur $\mathrm{PbO}_{2} 30$ ppi: $\mathrm{NaOH} 2 \mathrm{M}$; $\mathrm{K}_{3}\left[\mathrm{Fe}(\mathrm{CN})_{6}\right] 5 \cdot 10^{-2} \mathrm{M} ; \mathrm{K}_{4}\left[\mathrm{Fe}(\mathrm{CN})_{6}\right] 5 \cdot 10^{-3} \mathrm{M}$.

L'électrode de comparaison était constituée par un fil de $5 \cdot 10^{-3} \mathrm{~m}$ de diamètre, enroulé en spirale; comme anode, nous avons utilisé un fil de $\operatorname{Pt}\left(A=2.04 \cdot 10^{-4} \mathrm{~m}^{2}\right)$ et comme cathode un fil de nickel $\left(A=3,54 \cdot 10^{-4} \mathrm{~m}^{2}\right)$.

\subsection{Résultats et discussion}

La relation linéaire $\mathrm{Sh}=f(\mathrm{Re})^{\mathrm{a}}$ est observée pour les électrodes de comparaison (fig. 2) et pour les électrodes volumiques (fig. 3). La pente de ces droites est cependant un peu différente pour chaque electrode, ce qui signifie que soit le coefficient de transfert varie différemment avec la vitesse de l'électrolyte pour les différentes électrodes, soit que l'aire active varie avec la vitesse de l'électrolyte. En faisant l'hypothèse que $K_{\mathrm{d}}$, et $K_{\mathrm{d}}$ sontégaux, on peut calculer $A_{\text {e }}$ pour les différentes électrodes volumiques (fig. 4). Cette aire diminue avec l'augmentation de la vitesse de l'électrolyte dans le cas de la réduction du ferricyanure, alors qu'elle augmente légèrement dans le cas de l'oxydation du ferrocyanure sur $\mathrm{PbO}_{2}$. Les valeurs moyennes obtenues pour $V=10^{-2} \mathrm{~m} \mathrm{~s}^{-1}$ sont en bon accord avec celles mesurées par d'autres méthodes.

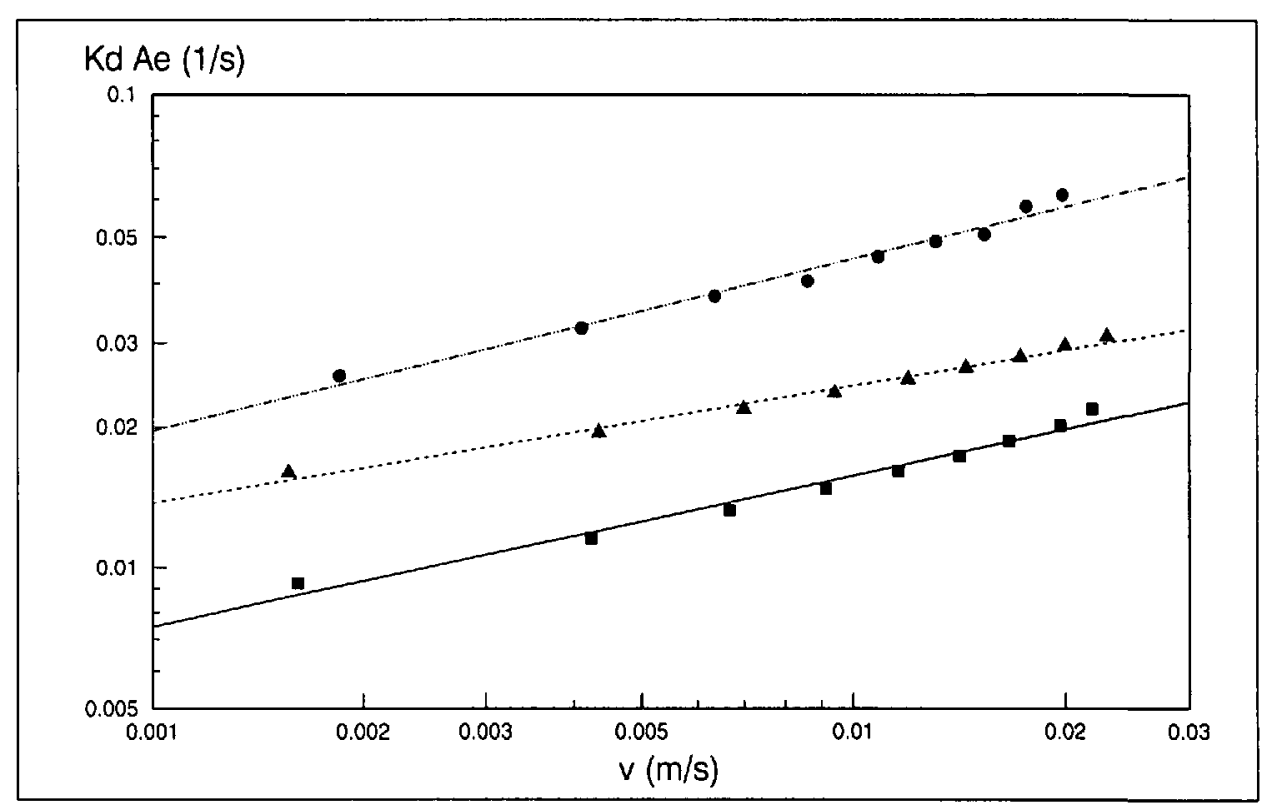

Fig. 3. Produit $\mathrm{K}$ A des différentes électrodes volumiques en fonction de la vitesse de l'électrolyte. $\cdots \mathrm{PbO}_{2} 30 \mathrm{ppi}$; pente: 0.36 ; FeII $\rightarrow$ FeIII; $\cdots \cdots \cdots$ Ni 20 ppi; pente: $0.2 \mathrm{~S}$; FeIII $\rightarrow$ Fell; $\longrightarrow$ PU 20 ppi; pente: 0.32; FeIII $\rightarrow$ FeII.

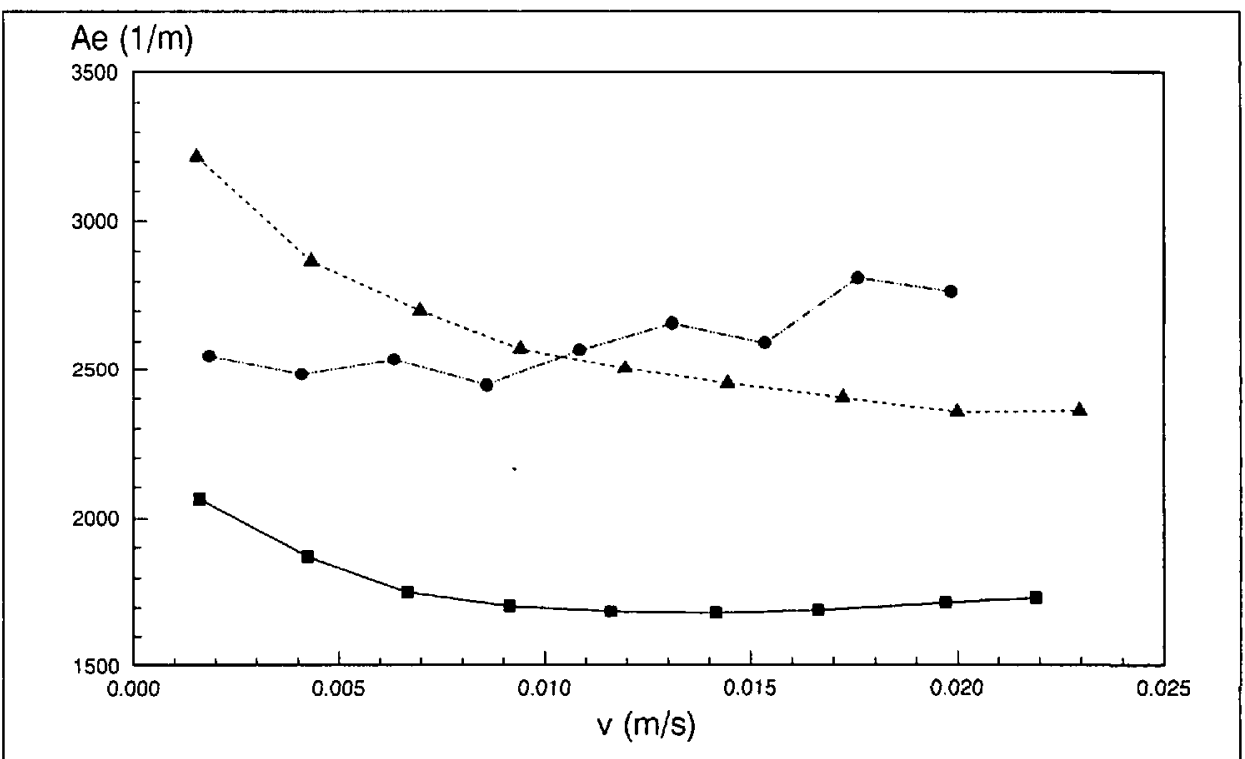

Fig. 4. Surface spécifique des différentes électrodes volumiques en fonction de la vitesse de

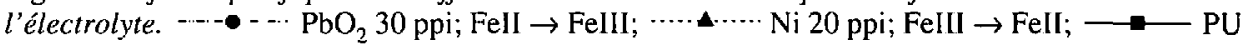
20 ppi; FeIII $\rightarrow$ FeII

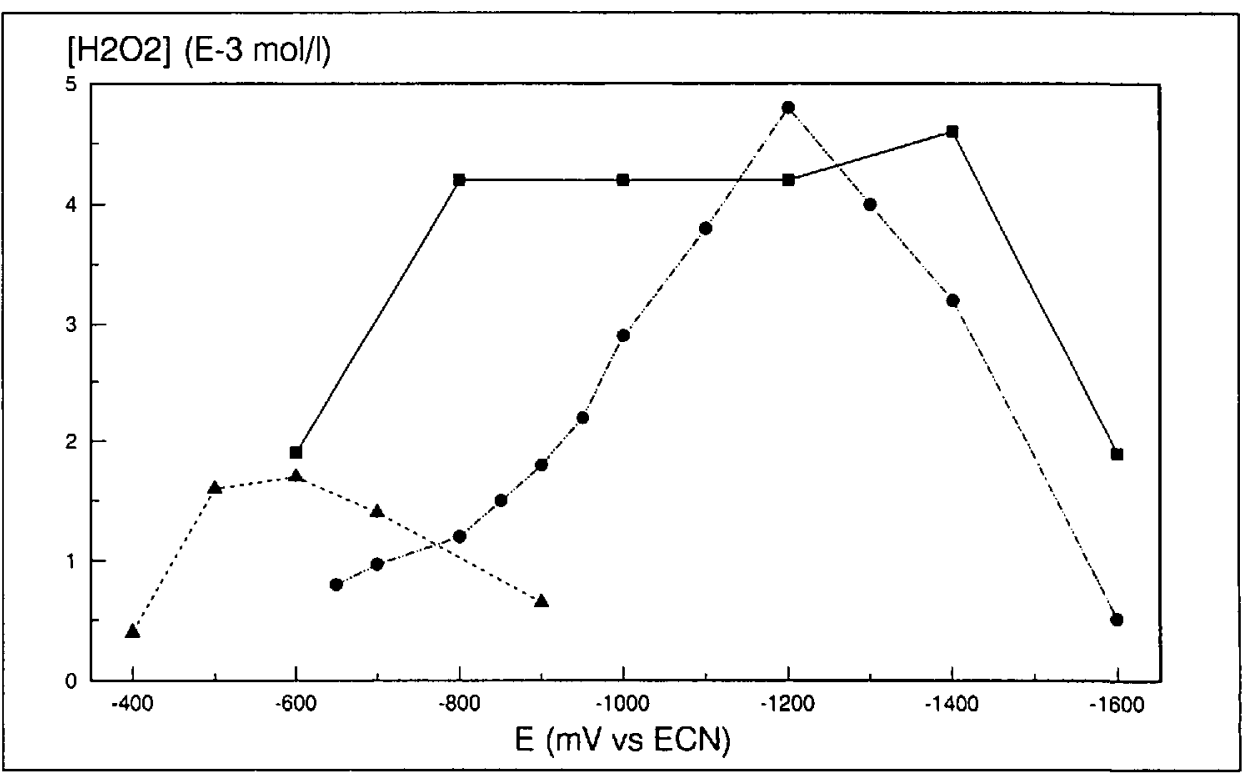

Fig. 5. Concentration en $\mathrm{H}_{2} \mathrm{O}_{2}$ obtenue après $3 \mathrm{~h}$ d'électrolyse à potentiel controlé. $\cdots \cdots \cdots \cdot \mathrm{RVCE}$ $\cdots \cdot-\mathrm{RVC} ; \longrightarrow$ RVCC 


\section{Applications des électrodes volumi- ques}

\subsection{Réduction de l'oxygène en eau oxy- génée}

La réduction électrochimique de l'oxygène en solution aqueuse se passe, en première approximation, en deux étapes selon la réaction globale 3

$\mathrm{O} 2 \stackrel{2 \mathrm{H}^{+}}{2 \mathrm{e}-} \mathrm{H}_{2} \mathrm{O}_{2} \underset{2 \mathrm{e}-}{\stackrel{2 \mathrm{H}^{+}}{\longrightarrow}} 2 \mathrm{H}_{2} \mathrm{O}$

La première réduction à deux électrons peut être électrocatalysée, en particulier par des groupements quinone-hydroquinone fixée à la surface de la cathode [6]

En raison de la faible concentration de l'oxygène dans les solutions aqueuses, le courant limite est toujours très faible; d'où l'intérêt évident d'utiliser une électrode volumique présentant une grande surface spécifique, afin d'obtenir une productivité élevée. On entend par productivitéla quantité de matière transformée par unité de temps et de volume du réacteur électrochimique. Nous avons utilisé une cellule cylindrique à deux compartiments séparés par une membrane en nafion. Le catholyte, un tampon phosphate à $\mathrm{pH}=8,5$ saturé en $\mathrm{O}_{2}$ à $25^{\circ}$, circule à travers la cathode avec une vitesse de $10^{-2} \mathrm{~ms}^{-1}$. Le volume total du catholyte est de $10^{-3} \mathrm{~m}^{3}$. Nous avons comparé trois cathodes différentes, chacune d'une aire de $\left.3710^{-2} \mathrm{~m}^{2}: a\right)$ un disque de RVC 30 ppi (RVC); $b$ ) un disque de RVC 30 ppi modifié électrochimiquement par 10 cycles de $+1,8 \mathrm{~V}$ à $-0,2 \mathrm{~V}$ à $5 \mathrm{mV} \mathrm{s}^{-1}$ (RVCE). Ce traitement crée des groupes quinone-hydroquinone à la surface du carbone [6] . c) un disque de RVC 30 ppi recouvert d'un film de poly $[N-(9,10-$ anthraquinone-2-carbonyl)ethylenimine [6] (RVCC). Ce film est déposé sur la mousse par immersion dans une solution du polymère dans du dichlorométhane.

La quantité d'eau oxygénée obtenue après $3 \mathrm{~h}$ d'électrolyse à divers potentiels contrôlés, est indiquée, pour chaque cathode, sur la fig. 5 .

On constate que les groupes quinonehydroquinone crés électrochimiquement (RVCE) catalysent la réduction à 2 électrons pour des potentiels de $-500 \mathrm{mV}$ environ; cependant la quantité d' $\mathrm{H}_{2} \mathrm{O}_{2}$ obtenue est très faible. L'activité électrocatalytique du polymère $(\mathrm{RVCC})$ est très nette entre -800 et $-1400 \mathrm{mV}$, par rapport à l'électrode non modifiée (RVC).

\subsection{Oxydation des ions $\mathrm{CN}^{-}$}

En milieu basique, l'oxydation électrochimique de l'ion $\mathrm{CN}^{-}$en cyanate peut être représentée par la réaction globale

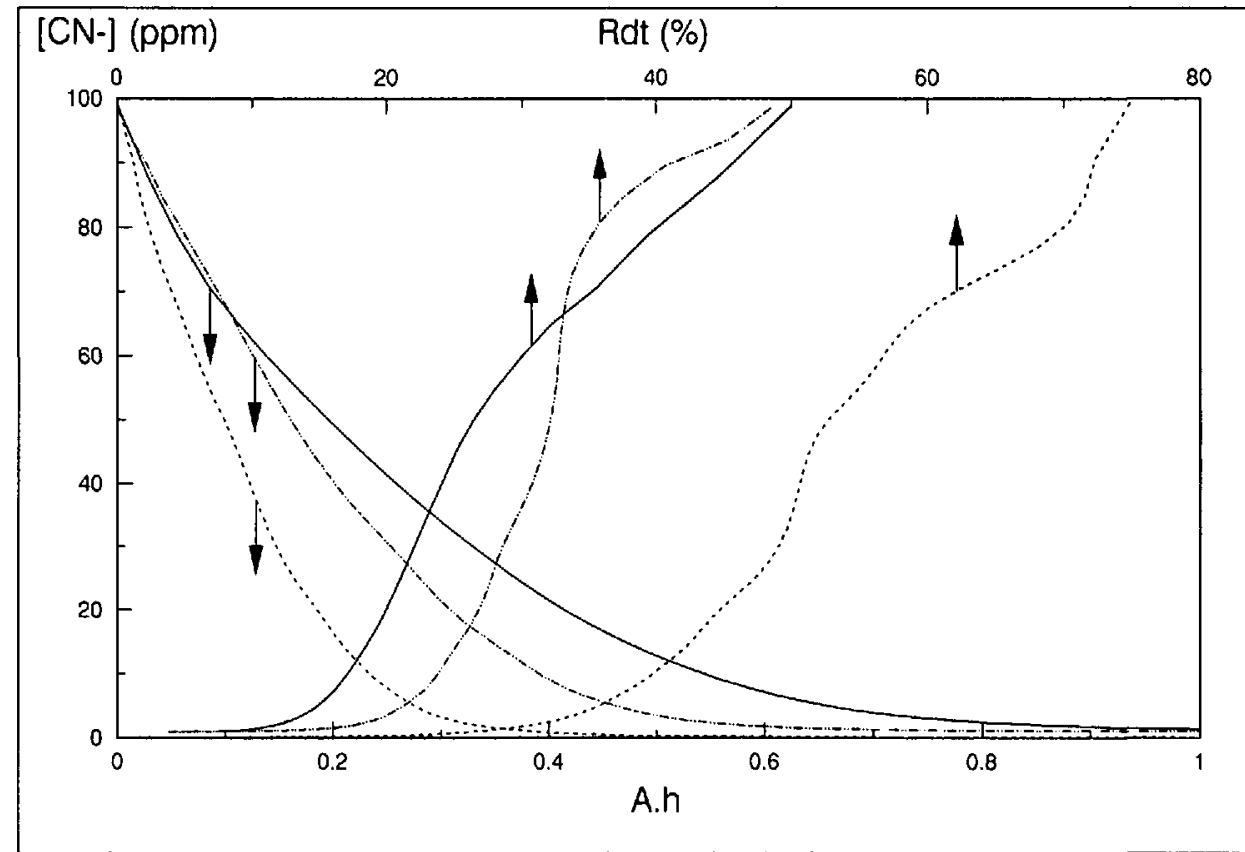

Fig. 6. Evolution de la concentration en $C N^{-}$pour differentes densités de courant en fonction du nombre d'Ah et évolution du rendement faradaïque en fonction de la concentration en $\mathrm{CN}^{-}$. $12,5 \mathrm{~A} \mathrm{~m}^{-2} ; \cdots \cdot \cdots, 2 \mathrm{~A} \mathrm{~m}^{-2} ; \cdots \cdots \cdots \cdots 3,11 \mathrm{~A} \mathrm{~m}^{-2}$

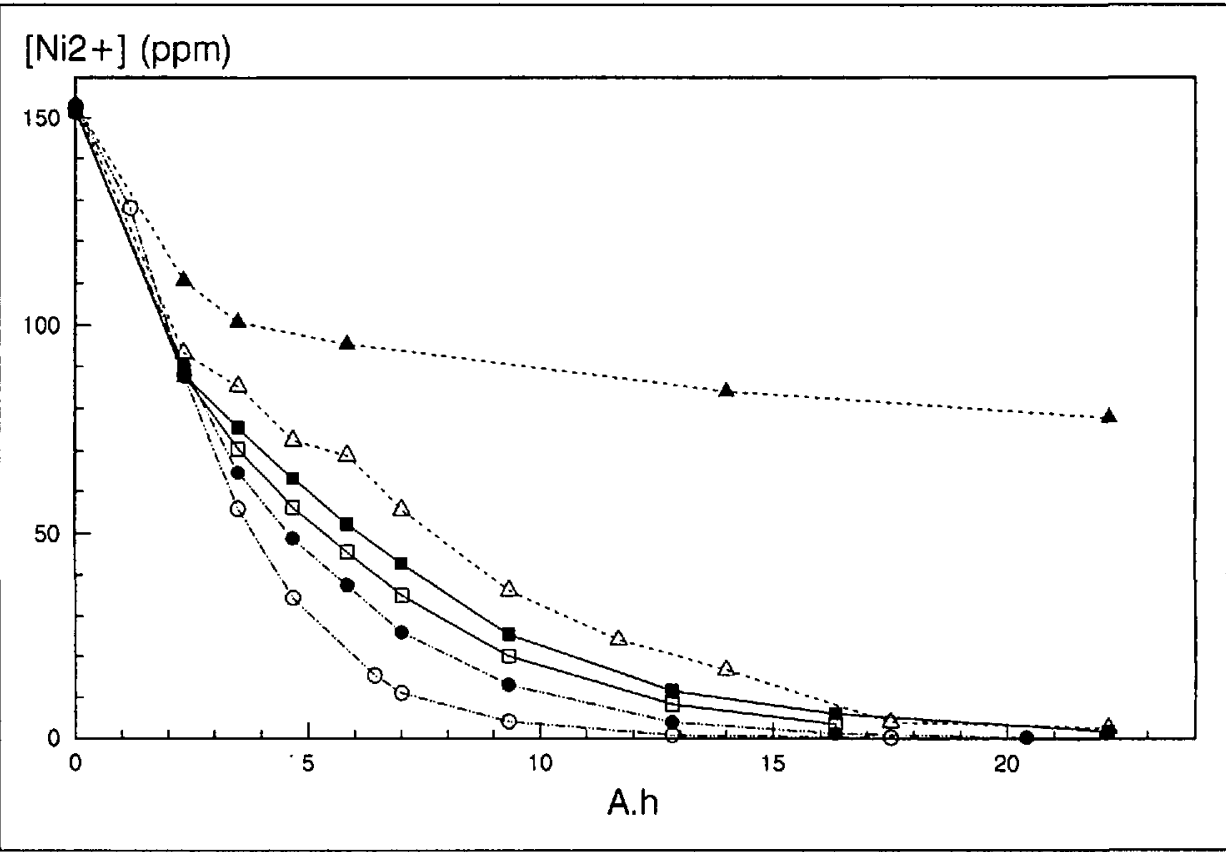

Fig. 7. Evolution de la concentration en $\mathrm{Ni}^{+2}$ en fonction du nombre d'Ah, en présence de divers acides carboxyliques. -.- - --- sans acides; __— acide glycolique; -..- - - acide lactique; … $\cdots$-... acide propionique, $\longrightarrow \square$ acide succinique; …...... acide citrique; densité de courant cathodique: $17,2 \mathrm{~A} \mathrm{~m}^{-2}$; débit de l'électrolyte: $0,156 \mathrm{~m}^{3} \mathrm{~h}^{-1}$; température: $25^{\circ}$.

$\mathrm{CN}^{-}+2 \mathrm{OH}^{-} \rightarrow \mathrm{OCN}^{-}+\mathrm{H}_{2} \mathrm{O}+2 \mathrm{e}^{-}$

Lorsque la concentration en $\mathrm{CN}^{-}$diminue, le rendement faradaïque devient très faible, et il convient de travailler avec de petites densités de courant anodique. L'utilisation d'électrode volumique permetd'atteindre un bon compromis entre une productivité élevée et un rendement faradaiique acceptable.

L'anode utilisée dans ce travail est constituée par une mousse de carbone vitreux réticulé (RVC $30 \mathrm{ppi}$ ) recouverte par ca. $40 \mathrm{mg} \mathrm{cm}^{-2}$ de $\mathrm{PbO}_{2}$ déposé élec- trochimiquement. Cette anode ne présente aucun signe de dégradation après plusieurs dizaines d'heures d'utilisation.

L'électrolyte, une solution tampon de carbonate de sodium à $\mathrm{pH} 10$ et $20^{\circ}$, contenant $100 \mathrm{ppm}$ de $\mathrm{CN}^{-}$, est percolé à travers les électrodes avec une vitesse linéaire de $4,4 \cdot 10^{-3} \mathrm{~m} \mathrm{~s}^{-1}$. Le volume total de l'électrolyte est de $500 \mathrm{ml}$ et la surface de l' anode est de $2.7 \cdot 10^{-2} \mathrm{~m}^{2}$. La variation de la concentration en cyanure est mesurée en continu avec une électrode spécifique $\mathrm{Ag} / \mathrm{AgI}$. Les résultats obtenus sont représentés sur la fig. 6 . 


\subsection{Réduction de $\mathrm{Ni}^{+2}$ en présence de complexants}

La réduction électrochimique de $\mathrm{Ni}^{+2}$ complexé par divers acides carboxyliques a été étudiée dans une cellule à cathode volumique de type PU 20 ppi. L'aire cathodique totale était de $0,26 \mathrm{~m}^{2}$ pour un volume de réacteur de $1,910^{-3} \mathrm{~m}^{3}$, ce qui correspond à un rapport aire cathodique/ volume du réacteur de $140 \mathrm{~m}^{-1}$. L'électrolyte est une solution $0,1 \mathrm{M}$ de $\mathrm{Na}_{2} \mathrm{SO}_{4}$ à $\mathrm{pH}$ 6 , contenant environ $100 \mathrm{ppm}$ de $\mathrm{Ni}^{+2}$ et $1 \%$ de divers acides carboxyliques. La vitesse linéaire de l'électrolyte percolant les électrodes est de $8 \cdot 10^{-3} \mathrm{~m} \mathrm{~s}^{-1}$. Une bonne agitation est assurée par de l'air introduit en bas de la cellule. Les résultats obtenus sont présentés sur la fig 7. On constate qu'en prolongeant la durée de la réduction, on peut atteindre une concentration en $\mathrm{Ni}^{+2}$ inférieure à $2 \mathrm{ppm}$ en pré- sence de tous les acides testés, à l'exception de l'acide citrique. La spéciation du Ni en présence d'acide citrique est actuellement étudiée par polarographie et au moyen d'une électrode spécifique.

Une partie de ce travail a été subventionnée par la Commission pour l'encouragement de la recherche scientifique (crédit CERS No. 1762.1).

Reçu le 20 janvier 1992

[1] Nomenclature: $I$ courant limite [A], $n$ : nombre d'électrons échangés, $F$ : constante de Faraday $\left[\mathrm{A} \mathrm{s}^{-1}\right], A$ : aire de l'électrode $\left[\mathrm{m}^{2}\right], K_{\mathrm{d}}$ coefficient de transfert de matière $\left[\mathrm{ms}^{-1}\right], c_{\mathrm{O}}$ concentration de l'espece élec- troactive dans l'électrolyte $\left[\mathrm{mol} \mathrm{m}^{-3}\right], A_{2}$ : aire spécifique par unité de volume $\left[\mathrm{m}^{2} \mathrm{~m}^{-3}\right.$ ou $\left.\mathrm{m}^{-1}\right], v$ : vitesse moyenne de l'électrolyte $\left[\mathrm{ms}^{-1}\right], L$ : longueur de l'électrode volumique dans le sens du flux [m]: $Q_{Y}$ : débit volumique de l'électrolyte $\left[\mathrm{m}^{3} \mathrm{~s}^{-1}\right]$, Sh: nombre adimensionnel de Sherwood: Re: nombre adimensionnel de Reynold.

[2] F. Coeuret, A. Storck, 'Eléments de Génie Electrochimique', Lavoisier, Paris, 1984

[3] (Ni): Sorapec, 192 Av. Carnot, F-94124 Fontenay-sous-Bois; (PU): Eltech, 18 Chemin Aulx, CH-1228 Plan-les-Ouates; (RVC): Energy Research and Generation 900 Stanford Avenue, Oakland, Ca. 94608. USA.

[4] S. Langlois, F. Couret, J. Appl. Electrochem. 1989, 19, 43.

[5] R.E. Sioda, J. Appl. Electrochem. 1978, \&, 297.

[6] Ch. Legrand, L.L. Miller, J. Electromal. Chem. 1981, 117, 267.

[7] T. Nagaoka, T. Yoshino, Anal. Chem. 1986, $58,1037$.
Chimia 46 (1992) 155-158

(C) Schweiz. Chemiker-Verband; ISSN 0009-4293

\title{
Complexes of Copper with a Flexible Bis-benzimidazole Ligand
}

\author{
Gérald Bernardinelliª), Anita Kübel-Pollak ${ }^{b}$ ), Stephane Rüttimann ${ }^{b}$ ), and Alan \\ F. Williams $\left.{ }^{b}\right)^{*}$
}

Abstract. The flexible bidentate ligand 1,3-bis(benzimidazol-2-yl)propane, $\mathrm{L}^{3}$, and its $N$-methylated derivative $\mathrm{L}^{4}$, form complexes with $\mathrm{Cu}^{\mathrm{I}}$ and $\mathrm{Cu}^{\mathrm{II}}$. The $\mathrm{X}$-ray crystal structure of $\left[\mathrm{Cu}\left(\mathrm{L}^{4}\right)(\mathrm{MeCN})\right]\left(\mathrm{PF}_{6}\right)(1)$ shows a trigonal coordination of the $\mathrm{Cu}^{1}$ with the eight-membered chelate ring adopting a half-chair conformation. With $\mathrm{Cu}^{\mathrm{Il}}$ in $\mathrm{EtOH}$ $\mathrm{L}^{3}$ forms dimeric $\left[\left(\mathrm{L}^{3}\right) \mathrm{Cu}(\mu-\mathrm{EtO})_{2} \mathrm{Cu}\left(\mathrm{L}^{3}\right)\right]\left(\mathrm{ClO}_{4}\right)_{2} \cdot 2 \mathrm{EtOH}(2)$ whose X-ray crystal structure shows $\mathrm{Cu}^{1 \mathrm{ll}}$ in a distorted square-planar environment with one bidentate ligand and two bridging ethoxides. The chelate ring now has a boat-chair conformation which forms a hydrophobic pocket around the metal.

\section{Introduction}

The formation of polynuclear complexes with unusual structural features such as double helices [1], triple helices [2], or knots [3] is a subject of current interest in coordination chemistry. Some years ago [4], we showed that the ligand 2,6-bis(1-methylbenzimidazol-2-yl) pyridine, $\mathrm{L}^{\mathrm{l}}$, forms with $\mathrm{Cu}^{\mathrm{l}}$ a double helical- dinuclear complex $\left[\mathrm{Cu}_{2}\left(\mathrm{~L}^{\mathrm{J}}\right)_{2}\right]^{2+}$, in which $\mathrm{L}^{1}$ acts as a bis-monodentate ligand, wrapping around the $\mathrm{Cu}-\mathrm{Cu}$ axis in such a way as to give a linear coordination of the $\mathrm{Cu}$ atom. The complex shows a weak bridging interaction due to the pyridines, and a strong stacking interaction between benzimidazoles in the two strands. If the pyridine of $L^{1}$ is replaced by a $P h$ group to give 1,3-bis(1-methylbenzimidazol-2-yl)ben- zene, $\mathrm{L}^{2}$, reaction with $\mathrm{Cu}^{1}$ also affords a dinuclear complex $\left[\mathrm{Cu}_{2}\left(\mathrm{~L}^{2}\right)_{2}\right]^{2+}[5]$, in which the $\mathrm{Cu}$-atom is still linearly coordinated, but the complex is now centrosymmetric, with the ligand adopting a different conformation in which a stacking interaction between the bridging $\mathrm{Ph}$ groups is possible. $\left[\mathrm{Cu}_{2}\left(\mathrm{~L}^{2}\right)_{2}\right]^{2+}$ may be regarded as a conformer of $\left[\mathrm{Cu}_{2}\left(\mathrm{~L}^{1}\right)_{2}\right]^{2+}$, since the two structures may be interconverted simply by twisting about $\mathrm{Cu}-\mathrm{N}$ and benzimidazole-Ph or benzimidazole-pyridine bonds. The double-helical structure of $\left[\mathrm{Cu}_{2}\left(\mathrm{~L}^{1}\right)_{2}\right]^{2+}$ is not possible for $\left[\mathrm{Cu}_{2}\left(\mathrm{~L}^{2}\right)_{2}\right]^{2+}$, since it would result in unacceptably short $\mathrm{Cu}-\mathrm{H}$ distances; it was, therefore, of interest to study the more flexible ligand 1,3-bis(benzimidazol-2yl)propane, $\mathrm{L}^{3}$, and its $N$-methylated derivative $\mathrm{L}^{4}$, which maintain the three $\mathrm{C}$ atom spacer between the benzimidazoles. but give two additional torsional angles of freedom. In this paper, we present the $X$ ray crystal structures of complexes of $\mathrm{L}^{3}$ and $\mathrm{L}^{4}$ with $\mathrm{Cu}^{\mathrm{II}}$ and $\mathrm{Cu}^{1}$, respectively.

\footnotetext{
*Correspondence: Dr. A.F. Williams ${ }^{b}$ )

a) Laboratoire de Cristallographie aux Rayons- $X$ 24, quai Emest-Ansermet $\mathrm{CH}-1211$ Genève 4

b) Département de Chimie Minérale. Analytique et Appliquée 30, quai Ernest-Ansermet $\mathrm{CH}-1211$ Geneve 4
} 